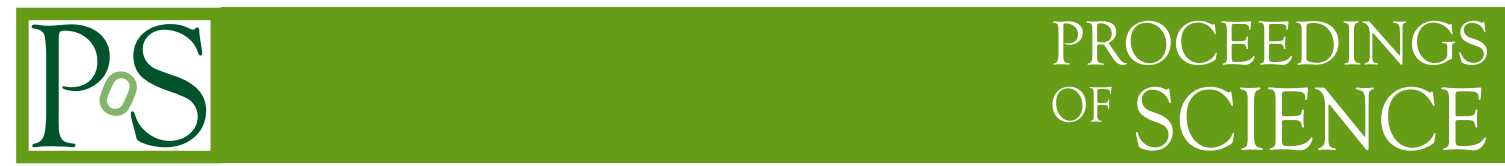

\title{
Search for a $D^{*} p$ resonance at HERA II
}

\author{
Katja KRUEGER * \\ Kirchhoff-Institut für Physik, Ruprecht-Karls-Universität Heidelberg \\ E-mail: kruegerk@mail.desy.de
}

The production of a possible narrow charmed baryonic resonance decaying into a $D^{\star}$ meson and a proton in deep inelastic scattering at HERA is investigated. The analysis is based on data taken with the H1 detector in the years 2004-2007, corresponding to an integrated luminosity of $\sim 348 \mathrm{pb}^{-1}$. No significant signal is found and an upper limit on the number of signal events is determined at $95 \%$ confidence level, taking into account only statistical errors.

European Physical Society Europhysics Conference on High Energy Physics

July 16-22, 2009

Krakow, Poland

*on behalf of the H1 Collaboration 


\section{Introduction}

Since the publication [1] of an excess in the $D^{\star} p$ mass spectrum around $3.1 \mathrm{GeV}$ in the HERA I data by the $\mathrm{H} 1$ collaboration this candidate for a charmed pentaquark state has been searched for by other experiments. So far no confirmation was found. The new HERA II data offer the possibility to look for this excess with the $\mathrm{H} 1$ detector in a larger independent data sample measured by the same detector.

\section{Event selection}

The data sample corresponds to an integrated luminosity of $\sim 348 \mathrm{pb}^{-1}$ and was recorded with the H1 experiment in the years 2004-2007, where either electrons or positrons with an energy of $27.6 \mathrm{GeV}$ were collided with $920 \mathrm{GeV}$ protons. The data are analysed in the photon virtuality range $2<Q^{2}<100 \mathrm{GeV}^{2}$ and in the inelasticity range $0.05<y<0.7$. Additional cuts are applied to restrict the analysis to the range where the detector is most efficient. Due to the detector changes during the HERA upgrade, these cuts reduce the phase space of the HERA II analysis at low $Q^{2}$ compared to the HERA I data, covering $\sim 80 \%$ of the total HERA I phase space.

$D^{\star}$ mesons are identified in their golden decay channel $D^{\star \pm} \rightarrow D^{0} \pi_{\text {slow }}^{ \pm} \rightarrow K^{\mp} \pi^{ \pm} \pi_{\text {slow }}^{ \pm}$. For the so called "wrong charge" (WC) background the kaon and pion have the same charge, such that these particles cannot origin from a $D$ meson, while the $\pi_{\text {slow }}$ must have the opposite charge. The tracks of the decay particles are reconstructed by the central tracking detector. The visible region, in which $D^{\star}$ mesons are reconstructed, is restricted to $p_{t}\left(D^{\star}\right)>1.5 \mathrm{GeV}$. In order to suppress the non-charm background the pseudo-rapidity is restricted to $-1.5<\eta\left(D^{\star}\right)<1.0$ and the inelasticity of the $D^{\star}$ meson, $z\left(D^{\star}\right)=\frac{\left(E-p_{z}\right)_{D^{\star}}}{2 y E_{e}}$, to $z\left(D^{\star}\right)>0.2$. In contrast to the published HERA I analysis, no particle identification by $\mathrm{d} E / \mathrm{d} x$ requirements is used for the $D^{\star}$ selection. The distribution of $\Delta M_{D^{\star}}=M\left(K \pi \pi_{\text {slow }}\right)-M(K \pi)$ is shown in Figure 1 (left). The number of $D^{\star}$ mesons, determined by a simultaneous fit to the signal and to the WC background in the $\Delta M_{D^{\star}}$ distribution, is $N\left(D^{\star}\right)=$ $15576 \pm 194$.
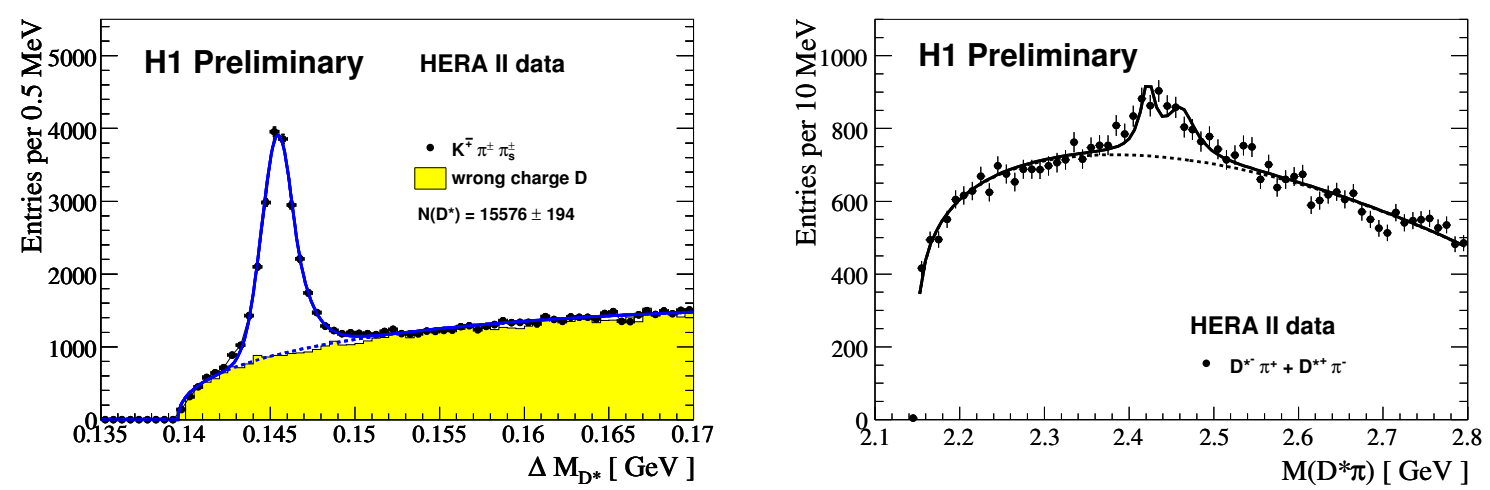

Figure 1: (left) The distribution of $\Delta M_{D^{\star}}$ for the signal sample (black points) and the wrong charge background (histogram). (right) The distribution of $M\left(D^{\star} \pi\right)$ in the $D^{\star}$ signal data sample. The data are fitted with the sum of two Breit-Wigner functions and a background function. 


\section{Results}

In order to show the sensitivity of the data to the decay of heavier charmed particles into a $D^{\star \pm}$ meson and an additional charged particle, the decay of the $D_{1}(2420)^{0}$ and the $D_{2}^{\star}(2460)^{0}$ into a $D^{\star}$ and a pion is studied. The pion candidate is required to have the opposite charge to the $D^{\star}$ and a transverse momentum larger than $0.12 \mathrm{GeV}$. The resulting mass distribution, reconstructed by $M\left(D^{\star} \pi\right)=M\left(K \pi \pi_{\text {slow }} \pi\right)-M\left(K \pi \pi_{\text {slow }}\right)+M_{D^{\star}}$ with $M_{D^{\star}}=2.010 \mathrm{GeV}$, is shown in Figure 1 (right). An enhancement is seen at the expected $D_{1}$ and $D_{2}^{\star}$ masses.

The selection of pentaquark candidates is kept close to the "high proton momentum" selection of the published analysis of HERA I data. The $\mathrm{d} E / \mathrm{d} x$ identification of protons is not used because the $\mathrm{d} E / \mathrm{d} x$ calibration of HERA II data is being finalised. Central tracks with opposite charge to the $D^{\star}$ meson and a momentum $p(p)>2 \mathrm{GeV}$ are combined with the $D^{\star}$ meson decay tracks to form a pentaquark candidate. The invariant mass $M\left(D^{\star} p\right)$ is calculated with the assumption of the proton mass by $M\left(D^{\star} p\right)=M\left(K \pi \pi_{\text {slow }} p\right)-M\left(K \pi \pi_{\text {slow }}\right)+M_{D^{\star}}$. The resulting mass distribution is shown in Figure 2 for HERA I and for HERA II data. The same kinematic cuts as described in the previous section have been applied to the HERA I data which correspond to an integrated luminosity of $84 \mathrm{pb}^{-1}$. While the HERA I data show the known clear peak structure at $3.1 \mathrm{GeV}$, nothing similar is found in the HERAII data. In addition to the correctly charged track combinations for $D^{\star} p$ candidates the WC $D^{\star}$ candidates are combined with oppositely charged proton candidates and shown as the light shaded histogram. To reduce fluctuations the full $\Delta M_{D^{\star}}$ histogram is taken and normalized to the number of events in the $D^{\star}$ mass region $(145.4 \pm 2.5 \mathrm{MeV})$. The sum of the wrong charged background and a $D^{\star} \mathrm{MC}$ simulation (dark histogram, normalised to number of $D^{\star}$ candidates in the $D^{\star}$ mass region) describes the right charge distribution reasonably well. Also the sum of HERA I and HERA II data shows no significant structures.
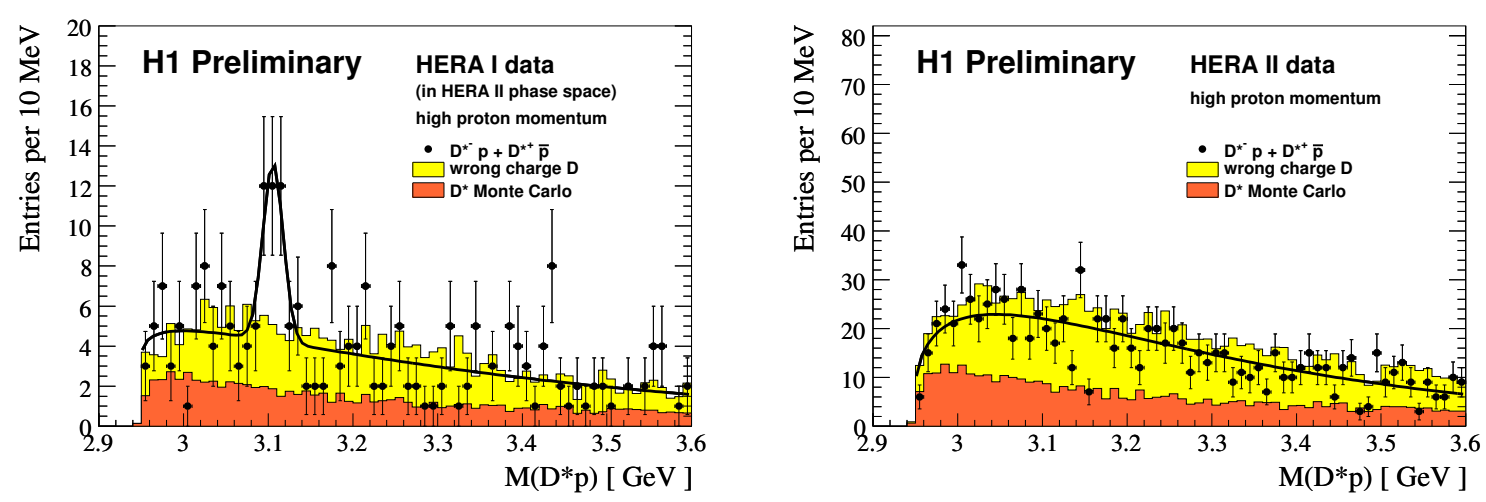

Figure 2: The distribution of $M\left(D^{\star} p\right)=M\left(K \pi \pi_{\text {slow }} p\right)-M\left(K \pi \pi_{\text {slow }}\right)+M_{D^{\star}}$ for the high proton momentum selection $(p(p)>2 \mathrm{GeV})$ in the HERA I (left) and HERA II (right) data samples. The right charge data sample is shown as black points, the background from a $D^{\star}$ Monte Carlo and the wrong charged $D^{\star}$ background as dark and light shaded histograms.

Under the assumption that a possible $D^{\star} p$ resonance has a Gaussian resolution of $12 \mathrm{MeV}$ at a mass of $3.1 \mathrm{GeV}$ (in agreement with the resolution in HERA I data), 16.3 events is determined as an upper limit on the number of signal events at the $95 \%$ CL, taking into account only statistical 
errors. This corresponds to a ratio of $D^{\star} p$ to $D^{\star}$ of 1.0 per mille. The corresponding ratio in the HERA I data sample with the same kinematic cuts is $8.1 \pm 2.1$ per mille.

As a cross check to whether the region of the enhancement in $M\left(D^{\star} p\right)$ contains a larger $D^{\star}$ fraction, the $\Delta M_{D^{\star}}$ distribution is studied for events in a $\pm 15 \mathrm{MeV}$ window around the HERA I $D^{\star} p$ signal and a sideband region, 2.99 to 3.07 and 3.13 to $3.21 \mathrm{GeV}$, scaled by a factor $3 / 16$ to account for the different widths (see Figure 3). While for HERA I the $\Delta M_{D^{\star}}$ distributions of the two regions differ around the expected value for $D^{\star}$ mesons, the signal and sideband distributions agree for HERA II data.
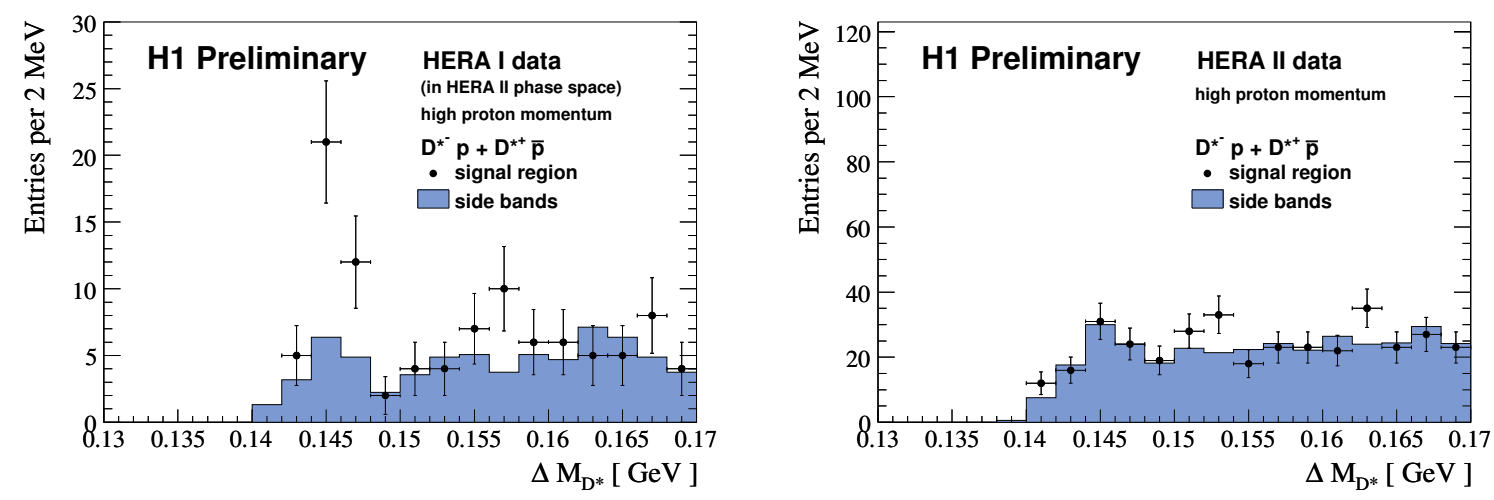

Figure 3: The $\Delta M_{D^{\star}}$ distribution in the HERA I (left) and HERA II (right) data samples for events in a $30 \mathrm{MeV}$ window around $3.1 \mathrm{GeV}$ in $M\left(D^{\star} p\right)$ compared to the corresponding distribution from the side bands (2.99 to 3.07 and 3.13 to $3.21 \mathrm{GeV})$ in the $M\left(D^{\star} p\right)$ distribution, normalised according to the widths of the chosen sample regions.

\section{Conclusion}

A study of the invariant mass spectrum of identified $D^{\star}$ meson candidates with high momentum proton candidates similar to the HERA I search for a narrow charmed baryonic resonance has been performed with HERA II data. The selection is kept close to the "high proton momentum" selection of the published HERA I analysis. The structure at $\sim 3.1 \mathrm{GeV}$ is reproduced in HERA I data in the kinematic range accessible in HERA II, which covers $\sim 80 \%$ of the HERA I phase space. In the HERA II data sample, which is roughly 4 times larger, no significant peak structure is visible. 16.3 events at a mass of $3.1 \mathrm{GeV}$ is determined as an upper limit on the number of signal events in HERA II data at the $95 \% \mathrm{CL}$, taking into account only statistical errors. This corresponds to a ratio of $D^{\star} p$ to $D^{\star}$ of 1.0 per mille. In the $\Delta M_{D^{\star}}$ distribution an enrichment at the expected value for $D^{\star}$ mesons is found in the $D^{\star} p$ "signal" region relative to the sidebands only for HERAI but not for the HERA II data sample.

\section{References}

[1] A. Aktas et al. [H1 Collaboration], Phys. Lett. B588 17 (2004). 